\title{
Robotic-assisted parathyroidectomy through lateral cervical approach: first results in Belgium
}

\section{S Van Slycke, K Van Den Heede, K Magamadov, N Brusselaers \& H Vermeersch}

To cite this article: S Van Slycke, K Van Den Heede, K Magamadov, N Brusselaers \& H Vermeersch (2019): Robotic-assisted parathyroidectomy through lateral cervical approach: first results in Belgium, Acta Chirurgica Belgica

To link to this article: https://doi.org/10.1080/00015458.2019.1693155

Accepted author version posted online: 18 Nov 2019.

Submit your article to this journal $₫$

Q View related articles $₫$

View Crossmark data \lceil 


\section{ROBOTIC-ASSISTED PARATHYROIDECTOMY THROUGH LATERAL CERVICAL} APPROACH: FIRST RESULTS IN BELGIUM.

VAN SLYCKE S, MD $D^{1,2,3}$ - VAN DEN HEEDE $K, M D^{1}-$ MAGAMADOV $K, M D^{1}-$ BRUSSELAERS $N, M D, P h D^{2,4,5}-$ VERMEERSCH H, MD, PhD ${ }^{5,6}$

Corresponding Author:

Sam Van Slycke

OLV Clinic Aalst, Belgium

Moorselbaan 164, 9300 Aalst, Belgium

003253724506

Email: dr.samvanslycke@gmail.com

Sam Van Slycke \& Klaas Van Den Heede equally contributed to this article as first author.

Funding and Conflicts of Interest: the authors have no conflicts of interest

Word count: 2280 words 


\section{$\underline{\text { ABSTRACT (word count: 181) }}$}

Objective: A parathyroidectomy has been the treatment of choice for primary hyperparathyroidism. Especially the improved imaging techniques have led to minimally invasive techniques. Aim of this study was to evaluate our experience with robot-assisted parathyroidectomy through lateral cervical approach.

Study Design: Prospective clinical cohort.

Methods: All consecutive patients who underwent a robotic-assisted parathyroidectomy for primary hyperparathyroidism since 2011 were enrolled in this study. Demographic data, biometrics, imaging data and surgery data were collected. Main outcomes were postoperative hypocalcemia, recurrent laryngeal nerve paralysis and other postoperative complications.

Results: Twenty-two patients were enrolled. When patients with conversion were excluded (39\%) mean operating time was 69 minutes. In all patients a normal value of serum PTH-levels was achieved 4 hours postoperatively. Mean value of serum calcium was $2,92 \mathrm{mmol} / \mathrm{L}$ preoperative and $2,33 \mathrm{mmol} / \mathrm{L}$ postoperative. There was no persistent hypocalcemia in any of our patients. $87 \%$ was discharged on the first postoperative day. Esthetic results were excellent.

Conclusions: Robotic-assisted parathyroidectomy through lateral cervical approach is a safe and feasible procedure in patients with posteriorly localized parathyroid adenomas. Preoperative imaging techniques are crucial to detect the exact location.

Keywords: primary hyperparathyroidism, parathyroidectomy, robotically assisted, lateral cervical approach 


\section{INTRODUCTION}

Primary hyperparathyroidism (pHPT) is a common disease in endocrinology, characterized by a disruption of the calcium metabolism. It typically presents itself with abnormally high levels of serum calcium and an unadapted high level of parathyroid hormone (PTH). Cause of this disorder is an autonomous hypersecretion of one or more parathyroid glands.

The disease can be entirely asymptomatic or produce somatic symptoms including nephrolithiasis, hypertension, bone pain, pathological fractures, abdominal pain, peptic ulcers, polyuria, polydipsia, neuromuscular pain or psychiatric disorders.

Diagnosis is based on biochemistry, with the following three elements: hypercalcemia, low phosphate and an unadapted PTH level. In 80 to $85 \%$ of the cases, a solitary parathyroid adenoma is responsible ${ }^{1}$.

The goal of treatment is to normalize the serum calcium levels, to relieve the symptoms and to avoid long-term complications of this disorder.

A parathyroidectomy has been the treatment of choice for a long time. Parathyroid surgery for primary hyperparathyroidism has changed completely since the first successful parathyroidectomy performed by Mandell in $1925^{2}$. Especially the improved imaging techniques to localize the parathyroid adenoma influenced the surgical strategy dramatically. In addition, during recent years, many new techniques were developed to perform a minimally invasive parathyroidectomy, e.g. minimal invasive open techniques ${ }^{3,4}$, gasless video-assisted ${ }^{5}$ and focused lateral endoscopic techniques ${ }^{6}$. A few studies also described a minimally invasive technique using a surgical robot $^{7-10}$, yet only through extra-cervical approach.

A robotic procedure following the lateral cervical approach as described by Henry et al. in 1999 (minimally invasive endoscopy $)^{6}$ has not yet been described. Since we applied this minimally invasive endoscopic parathyroidectomy already several times (on 23 cases with primary 
hyperparathyroidism), our objective was to evaluate our results in a prospective cohort study. Our experience in Belgium concerning the robot-assisted parathyroidectomy, a technique offered to some patients with a unilateral posteriorly localized parathyroid adenoma, confirmed by concordant results on ultrasonography and SPECT-CT is described.

\section{MATERIALS AND METHODS}

DATA

A prospective cohort study that evaluated the technique of the robot-assisted parathyroidectomy was conducted. This single center study was performed from September 2011 to December 2013. Ethical approval was obtained from the ethical committee of the Onze-Lieve Vrouw (OLV) Clinic, Aalst and all patients provided written informed consent. Neither financial support nor any free devices were received from the industry.

All consecutive patients who underwent a robotic-assisted parathyroidectomy for primary HPT since September 2011 were enrolled in this study. All procedures were performed by the same experienced endocrine surgeon (SVS).

\section{PATIENT SELECTION}

After initial diagnosis of PHPT through blood analysis, a thorough preoperative assessment with medical imaging was performed. At baseline (before surgery), levels of parathyroid hormone (PTH), phosphate, calcium, calcitonin, creatinin and 25-hydroxy vitamin D were measured, and all patients underwent ultrasonography and scintigraphy and/or SPECT CT. A 24-hour calciuria was performed to exclude benign familial hypercalcemic hypocalciuria. Calcitonin measurements and urinary catecholamines and normetanephrines were routinely performed to exclude MEN-syndrome 
(multiple endocrine neoplasia). Detailed information regarding the patient's demographic data and biometrics were collected.

The parathyroid adenomas were localized preoperatively, although these localization techniques have no influence on the diagnosis of primary hyperthyroidism. Nevertheless, it is very useful for the decision making of which surgical technique is the best option.

The suspicion of a solitary adenoma can be calculated with the CaPTHus-score, which uses 5 variables to calculate the probability. A total serum calcium level of $>12 \mathrm{mg} / \mathrm{dl}$, a PTH level of two or more times the upper limit of normal values, an ultrasound or sestamibi scan with evidence of a solitary adenoma and concordant information between ultrasonography and sestamibi scan. Every criterion is given one point when positive. A score of three or more gives a high probability of a solitary adenoma, with a positive predictive value of $100 \%{ }^{11,12}$.

Patients with concomitant findings on medical imaging showing a solitary posteriorly localized parathyroid adenoma were selected for a robotic-assisted parathyroidectomy. There were no specific exclusion criteria.

\section{OPERATING TECHNIQUE}

Parathyroidectomy was performed according to the technique of Henry ${ }^{6}$ but by means of a surgical robot (type Da Vinci® ${ }^{\circledR}$ SI surgical system (Intuitive Surgical Inc., Sunnyvale, CA, USA)). This operation requires general anesthesia. An 8,5mm incision is made laterally in the neck on the anterior side of the sternocleidomastoid muscle. Through this incision, a dissection is performed, providing an access medially of the carotid artery and laterally of the strap muscles. Through two extra $5 \mathrm{~mm}$ incisions, two working trocars were placed 3 to $4 \mathrm{~cm}$ cranially and caudally from the central incision. Consequently, the optical trocar is placed and fixated around the central incision. To obtain good visibility during the whole procedure, $\mathrm{CO}_{2}$ is insufflated up to a constant pressure of $8 \mathrm{mmHg}$. The robotic instruments are consequently docked under endoscopic control: a grasper is placed in the 
left arm of the robot and a monopolar cautery hook is placed in the right arm. Through blunt dissection, the adenoma can be freed from the surrounding structures and the vascular pedicle is cut by coagulation. The trocars and the adenoma are then removed, and the skin is closed. The resection specimen is transported dry to the pathological laboratory, where a frozen section procedure is performed.

Right before and during the operation, the value of PTH is followed on baseline moments described in the NACB guidelines ${ }^{13}$ with detection in the blood taken by the anesthesiologist through an intravenous line in the great saphenous vein, or through an arterial line placed in the radial artery. These moments are with intubation (T1), with incision (T2), just before resection of the adenoma (T3), 5 minutes after resection (T4), 15 minutes after resection (T5) and optionally 30 minutes after resection (T6).

Frozen section and intraoperative PTH measurement with more than $50 \%$ decrease 15 minutes after resection in comparison of the highest baseline level of the patient (MIAMI-criteria) are the criteria for surgical success and demonstrate that there is no need of further exploration of the other parathyroid glands ${ }^{14}$.

Perioperative findings such as loss of blood, need for drainage, conversion and duration of surgery were documented as well.

\section{OUTCOMES}

Following surgery, the presence of hypocalcemia was documented. Parathyroid hormone (PTH) levels were recorded at 4 hours and 24 hours. Calcium levels were recorded on day 1.

Patients were discharged from hospital and follow-up was conducted on-site at 6 weeks postsurgery. Patients with persistent hypocalcemia lasting over 6 weeks were monitored until recovery of parathyroid function was achieved. Nerve injury/vocal cord assessment was performed using routine laryngoscopy during a pre-operative surgical visit and at 1 day after surgery. Patients with recurrent 
paralysis were monitored by laryngoscopy every 3 months until they had regained mobility, or up to a year after surgery. Permanent palsy is diagnosed when present 1 year after surgery.

Main outcomes were post-operative hypoparathyroidism (present or absent, defined as PTH < $15 \mathrm{pg} / \mathrm{dL}$ at four hours after the operation), post-operative recurrent nerve paralysis (present or absent) and other post-operative complications, classified according to the Clavien-Dindo system (categorized as no complications - Clavien score 0; or complications - Clavien score 1-3).

\section{$\underline{\text { RESULTS }}$}

This study enrolled 23 patients who underwent a robotic-assisted parathyroidectomy between September 2011 and October 2013. Patient characteristics are presented in Table 1.

Nine patients (39\%) were converted to a (small) cervicotomy. Two patients were converted because of adhesions due to previous surgery, two patients because of bleeding during surgery, one patient because of a gland incrusted in the thyroid lobe and three patients because of difficult dissection and bad insufflation. In one patient the adenoma could not be localized because of its anterior position. One patient underwent a wound exploration immediately after extubation because of a discrete neck hematoma due to diffuse small venous bleedings.

In three of these converted patients (13\%) a small drain was left for one day. Drainage wasn't necessary in the fully robotically assisted parathyroidectomy procedures.

Twenty out of twenty-three patients (87\%) were discharged on the first postoperative day, two patients on the second and one on the seventh postoperative day, because of multiple comorbidities and social reasons.

Perioperative characteristics are shown in Table 2.

All resection specimens were confirmed as a parathyroid gland adenoma on pathological protocol. 
In all 23 patients a normal value of serum PTH-level was achieved 4 hours postoperatively, as shown in Table 3.

Without exclusion of the converted patients, median follow-up time was 51 days (17-150). Mean operating time was 88 minutes (40-225). The mean value of serum calcium was $2,94 \mathrm{mmol} / \mathrm{L}(2,63-$ $3,60)$ preoperative and $2,37 \mathrm{mmol} / \mathrm{L}(1,93-2,63)$ postoperative, with a normal range between 2,15 $2,58 \mathrm{mmol} / \mathrm{L}$.

When patients with conversion were excluded, median follow-up time was 51 days (39-102). Mean operating time was 69 minutes (40-120). The mean value of serum calcium was $2,92 \mathrm{mmol} / \mathrm{L}(2,63-$ $3,60)$ preoperative and $2,33 \mathrm{mmol} / \mathrm{L}(1,93-2,55)$ postoperative, with a normal range between 2,15 $2,58 \mathrm{mmol} / \mathrm{L}$.

There was no persistent hypocalcemia in any of our patients. There was one patient who developed a permanent recurrent nerve paralysis (4,3\%).

Comparative results are shown in Table 4.

In short-term follow up, all patients but one revealed normal calcium levels. One of the converted patients underwent a re-operation because of persistent elevated PTH and serum calcium levels. Diagnosis of tertiary hyperparathyroidism was made, and she underwent a subtotal parathyroidectomy through cervical incision three months after the robot-assisted operation. Esthetic results were excellent (Fig. 2). No major complications occurred. One patient died during follow-up because of complications of a neurosurgical intervention. 


\section{DISCUSSION}

Considering the wide range of existing techniques for minimal invasive parathyroid surgery, there still remains a small margin for improvement in the lateral endoscopic approach. When compared to the technique described by Henry ${ }^{6}$, we believe that the use of the Da Vinci ${ }^{\circledR}$ Surgical System adds several benefits to the meticulous dissection required when operating in the cervical region. The ability to generate a high definition three-dimensional visual environment combined with a stable camera platform enhances the visibility of the recurrent laryngeal nerve (RLN), and parathyroid glands. Another advantage is the articulation of the instruments, which makes it possible to manipulate the parathyroid gland from more directions compared to the endoscopic straight instruments. Laundry et al. showed that in his transaxillary approach for the removal of thyroid and parathyroid glands, the endo-wrist articulation provides superior range of motion when compared to instruments used in standard fixed endoscopy ${ }^{8}$. The combination of these additional benefits facilitate the dissection, even in difficult cases where the adenoma is adherent to the thyroid gland.

Robotic assisted parathyroidectomy through a lateral cervical approach has shown to be a safe and feasible procedure in patients with a posteriorly localized parathyroid adenoma.

To date, there are still some disadvantages of the robotic approach. First of all, there is a bigger cost to perform the robotic technique. In our institution, this extra cost has not yet been passed on to the patient in any way in case of robotic parathyroid surgery.

Secondly, we have seen a longer mean operating time in the robotic procedure (88 minutes) compared to the standard endoscopic parathyroidectomy according to Henry ${ }^{6}$ (46 minutes) we performed in 27 cases. We also noticed a high conversion rate (up to $39 \%$ ). When our converted cases are left out of the analysis, our operation time was already remarkably lower (69 minutes). We believe that we are still in a learning curve, and we expect this difference to diminish with growing experience. The assumption is often made that the installation of the robotic system is very time consuming, but we can refute this assumption. In a well-organized operation theatre, the three 
components of the robot can already be positioned before the procedure. While the patient is brought under general anesthesia, the sterile drapes of the robot arms can already be put in place. Installation of the patient is parallel to the standard endoscopic approach described by Henry ${ }^{6}$. By the moment the operating time starts, the robot arms can be rolled in ready to perform, with a time loss of less than 30 seconds.

Third difference is the total length of skin incision. In the technique described by Henry ${ }^{6}$, two $3 \mathrm{~mm}$ incisions are made, and one $10 \mathrm{~mm}$ incision. In our technique, the two working port incisions are 5 $\mathrm{mm}$, which brings the total incision length to at least $18.5 \mathrm{~mm}$. Compared to our standard endoscopic approach, this is an augmentation of $2.5 \mathrm{~mm}$ more scare, which seems to diminish the cosmetic advantage of minimal invasive surgery. Nevertheless, as already has been stated before: minimally invasive parathyroid surgery is not only a question of length of the incision. All patients were satisfied about the cosmetic result, and the residual scars of the two $5 \mathrm{~mm}$ ports were practically invisible one month postoperative (Fig. 2). Hopefully, the further development of the robotic instruments may even provide us with smaller working tools, which reverses this issue.

\section{CONCLUSIONS}

Robotic-assisted parathyroidectomy through a lateral cervical approach has shown to be a safe and feasible procedure in patients with a posteriorly localized parathyroid adenoma. Our first results show a definitive cure in all of our patients.

We have to emphasize the importance of preoperative imaging techniques. It is essential to detect the exact location with preoperative imaging and verifying that it is a posterior localized solitary adenoma. Thanks to the measurements of the intraoperative PTH levels, it is possible to predict cure. When there is no concordant imaging, the intraoperative PTH level detection certainly is an important feature. 
The robotically assisted parathyroidectomy, a technique with a long learning curve, is nowadays offered to all patients who present with a unilateral posteriorly localized parathyroid adenoma, confirmed by concordant results on ultrasonography and SPECT-CT. It has become part of our routine practice.

In this study we did not compare our results of the robotically assisted approach with the totally endoscopic approach. Regarding this technique there are no randomized controlled trials available to date. 


\section{$\underline{\text { TABLES AND FIGURES }}$}

TABLE 1. Patient characteristics of the study cohort.

Age, N (\%)

45-59 years $\quad 11(47,8 \%)$

$60-85$ years $\quad 12(52,2 \%)$

Gender, N (\%)

$\begin{array}{ll}\text { Male } & 4(17,4 \%) \\ \text { Female } & 19(82,6 \%)\end{array}$

Body mass index, $\mathbf{N}(\%)$

$<20 \mathrm{~kg} / \mathrm{m}^{2}$

$2(8,7 \%)$

20.0-24.9 $\mathrm{kg} / \mathrm{m}^{2}$

$8(34,8 \%)$

$25.0-29.9 \mathrm{~kg} / \mathrm{m}^{2}$

$10(43,5 \%)$

$\geq 30.0 \mathrm{~kg} / \mathrm{m}^{2}$

$3(13,0 \%)$

Preoperative blood results

Calcemia ( $\mathrm{mmol} / \mathrm{L})$

$2,94(2,63-3,60)$

Phosphor ( $\mathrm{mmol} / \mathrm{L})$

$0,74(0,55-1,63)$

PTH (ng/L)

$218,2(75-875,8)$

25-OH Vit. $D(\mu \mathrm{g} / \mathrm{L})$

$23,18(6-46)$

Creatinin ( $\mathrm{mg} / \mathrm{dL}$ )

$0,98(0,5-3,03)$

Albumin ( $g / L)$

$43,2(33-50,6)$ 
TABLE 2. Perioperative characteristics of the study cohort.

Year of surgery, $\mathrm{N}(\%)$

$\begin{array}{ll}2011 & 8(34,8 \%) \\ 2012 & 10(43,5 \%) \\ 2013 & 5(21,7 \%)\end{array}$

Duration of surgery (minutes)

Median

Missing, $N(\%)$

Conversion, $\mathbf{N}(\%)$

Yes

Drainage, $\mathbf{N}(\%)$

Yes

Days of drainage (days)

Median

Missing

Weight specimen (milligrams)

Length of hospitalisation (days)
$9(39,1 \%)$ $3(13,0 \%)$

$88(40-180)$

$4(17,4 \%)$

$1(1-1)$

$1(33 \%)$

$1051(40-2640)$

$1(4,3 \%)$

$1,3(1-7)$ 
TABLE 3. Parathyroid hormone levels on baseline moments as described in the NACB guidelines.

\begin{tabular}{|c|c|c|c|c|c|c|c|c|}
\hline Patient Nr & $\mathrm{T1}$ & T2 & T3 & T4 & T5 & T6 & $T+4 h$ & $T+24 h$ \\
\hline 1 & 88,4 & 84,4 & 37,5 & 30,3 & I & I & 17,5 & 26,3 \\
\hline 2 & 73,7 & 81,6 & 45,3 & 28,4 & 15,9 & I & 8,8 & 8,3 \\
\hline 3 & 77,3 & 73,4 & 70,1 & 35 & 18,4 & 1 & 13,4 & 19,4 \\
\hline 4 & I & 86,4 & 86,4 & 33,3 & 33,5 & 38,3 & 26,1 & 51 \\
\hline 5 & 151,1 & 108,7 & 113,5 & 55,3 & 40 & I & 22,8 & 21,6 \\
\hline 6 & 65,9 & 62,8 & 17,1 & 32 & 22,2 & I & 10,4 & 16,5 \\
\hline 7 & 97,8 & 106,2 & 147,5 & 113,5 & 69,6 & I & 22,9 & 41,7 \\
\hline 8 & 93 & 93,5 & 48,3 & 39,4 & 35,9 & 38,3 & 32,8 & 46,4 \\
\hline 9 & 548,2 & 295,1 & 272,6 & 135 & 82,5 & I & 9,5 & 9,3 \\
\hline 10 & 676,7 & 681,4 & 666,1 & 677 & 996,5 & 409,6 & 92,1 & 14,4 \\
\hline 11 & 85,37 & 59,82 & 84,15 & 36,67 & 26,98 & 20,98 & 20,9 & 20,5 \\
\hline 12 & 448,6 & 435,1 & 413,7 & 1 & I & 1 & 336,8 & 320,9 \\
\hline 13 & 499,7 & 513,8 & 273,9 & 170 & 103 & I & 36,9 & 34,6 \\
\hline 14 & 102 & 92,3 & 43,1 & 24,4 & 18,3 & 12,2 & 12,2 & 27,7 \\
\hline 15 & 245,9 & 251,3 & 101,9 & 50,5 & 33,4 & 23,4 & 14,6 & I \\
\hline 16 & 86,9 & 92,4 & 28,5 & 17,5 & 10,5 & 14 & 8,6 & 20,5 \\
\hline 17 & 133,4 & 187,5 & 5,16 & 22,2 & 53 & 18 & 9,2 & 10,4 \\
\hline 18 & 98,4 & 78,9 & 39,8 & 28,7 & 17,2 & 18 & 19,0 & 25 \\
\hline 19 & 200,5 & 197,4 & 88 & 55,4 & 30,5 & 15,6 & 7,9 & 5,6 \\
\hline 20 & 99 & 92 & 45 & 1 & 13 & I & 9,5 & 14,4 \\
\hline 21 & 193,8 & 219,5 & 254,4 & 155 & 62,3 & 38,3 & 13,6 & 16,9 \\
\hline 22 & 97,8 & 96 & 146,2 & 144,8 & 1 & 1 & 33,9 & 12,4 \\
\hline 23 & 558,5 & 483,7 & 252,1 & 79,7 & 46,8 & I & 14,6 & 9,3 \\
\hline
\end{tabular}


TABLE 4. Comparative results between the total study cohort and a subgroup without the converted operations.

WITHOUT EXCLUSION OF CONVERTED OPERATIONS

Follow-up time (days)

Temporary hypocalcaemia, N (\%)

Recurrent nerve paralysis, $\mathrm{N}$ (\%)

Clavien Score

\section{WITH EXCLUSION OF CONVERTED OPERATIONS}

Follow-up time (days)

Median $51(17-150)$

Median $51(39-102)$

Temporary hypocalcaemia, $\mathbf{N}(\%)$

Yes $\quad 5(35 \%)$

Recurrent nerve paralysis, $\mathbf{N}(\%)$

Yes $\quad 1(4,3 \%)$

Yes

$1(7,1 \%)$

Clavien Score

$\begin{array}{ll}0 & 19(82,6 \%) \\ 1 & 4(17,4 \%) \\ 2 & / \\ 3 a / b & /\end{array}$

0

2) /

$3 a / b$
$1 \quad 3(21,4 \%)$ 
FIGURE 1. Robotic assisted parathyroidectomy using a Da Vinci® ${ }^{\circledR}$ surgical robot.

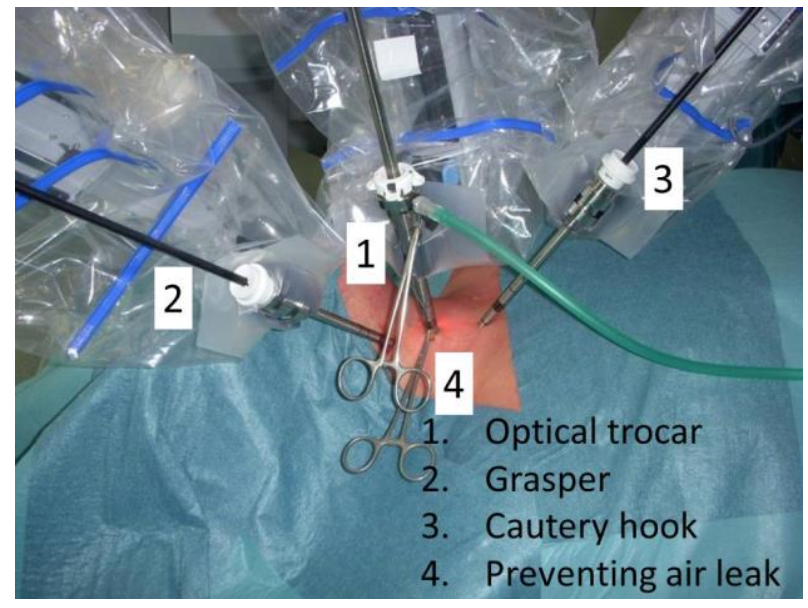

FIGURE 2. Satisfying cosmetic result one month after total endoscopic robotic parathyroidectomy, showing only a residual scar of the $10 \mathrm{~mm}$ incision. The two lesser incisions are almost invisible. The redness is due to a recent clinical examination.

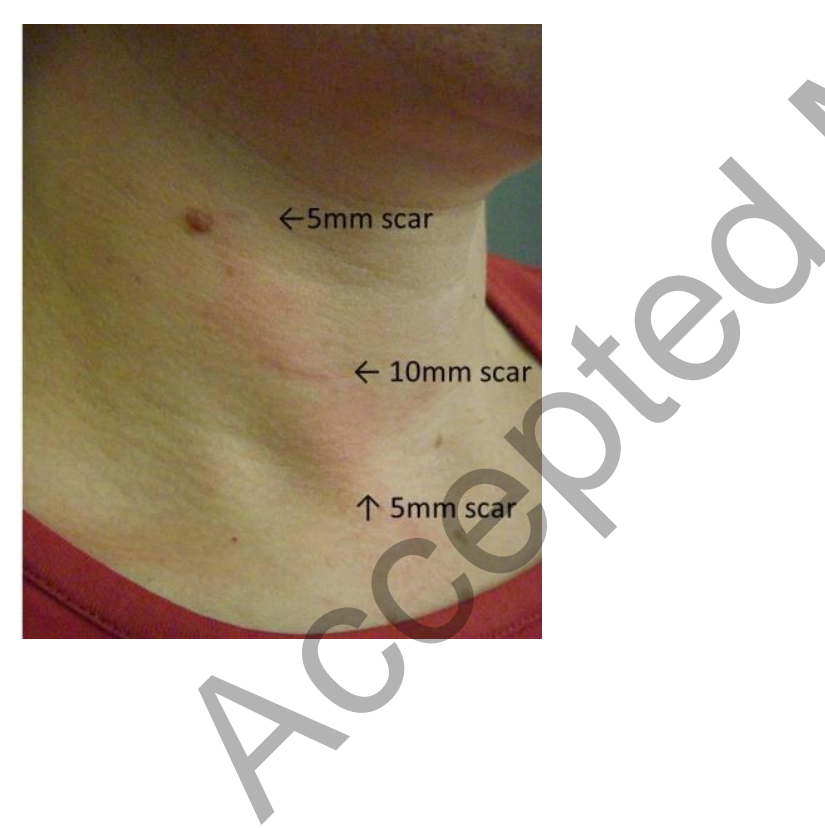




\section{REFERENCES}

1. Suliburk JW et al., Primary Hyperparathyroidism. Oncologist 2007; 12: 644-653

2. Delbridge LW et al., First parathyroid surgeon: Sir John Bland-Sutton and the parathyroids. ANZ journal of surgery 2007;77:1058-61

3. Wong $\mathrm{W}$ et al., Simplified minimally invasive parathyroidectomy: a series of 100 cases and review of the literature. Ann R Coll Surg Engl. 2011; 93(4):290-293

4. Udelsman $\mathrm{R}$ et al., One hundred consecutive minimally invasive parathyroid explorations. Ann Surg. 2000; 232(3): 331-339

5. Miccoli P et al., Minimally invasive video assisted parathyroidectomy (MIVAP). Eur J Surg Oncol. 2003; 29(2): 188-190

6. Henry JF et al., Endoscopic parathyroidectomy via a lateral neck incision. Ann Cir. 1999; 53(4): 302-306

7. Bodner J et al., Mediastinal parathyroidectomy with the da Vinci robot: presentation of a new technique. J Thorac Cadiovasc Surg., 2004; 127(6): 1831-1832

8. Profanter C et al., Robot-assisted mediastinal parathyroidectomy. Surg Endosc. 2004; 18(5): 868-870

9. Landry C et al., Robot assisted transaxillary surgery (RATS) for the removal of thyroid and parathyroid glands. Surgery 2011; 149(4): 549-555

10. Katz L et al., Robotic-assisted transaxillary parathyroidectomy of an atypical adenoma. Minim Invasive Ther Allied Technol. 2012; 21(3): 201-205

11. Fouquet et al., Totally endoscopic lateral parathyroidectomy: prospective evaluation of 200 patients. Langenbecks Archives of Surgery 2010; 395:935-940

12. Kebebew E et al., Predictors of Single-Gland vs Multigland Parathyroid disease in Primary Hyperparathyroidism. A Simple and Accurate Scoring Model. Arch Surg. 2006; 141:777-782

13. Nichols JH. et al., National Academy of Clinical Biochemistry Laboratory Medicine Practice Guidelines: Evidence Based Practice for Point of Care Testing. Clin Chim Acta 2007; 379(1-2):14-28

14. Carneiro D et al., Comparison of intraoperative iPTH assay (QPTH) criteria in guiding parathyroidectomy: which criterion is the most accurate? Surgery 2003; 134:973-979 